\title{
An Information Privacy Taxonomy for Collaborative Environments
}

\begin{abstract}
Purpose: Information Privacy is becoming an increasingly important field of research with many new definitions and terminologies. Along similar rates of increase are the use, uptake and expansion of Collaborative Environments. There is a need for a better understanding and classification of information privacy concepts and terms. The purpose of this paper is to provide a taxonomy of Information Privacy in Collaborative Environments. The knowledge provided from an information privacy taxonomy can be used to formulate better information privacy policies, practices, and privacy enhancing technologies (PET's).

Approach: Through the hierarchical classification and categorization of information privacy concepts and principles an organized representation of these components has been produced. Each area was well surveyed and researched and then classified into a number of sub-categories according to their nature and relevance.

Findings: A taxonomy was successfully developed with the identification of three high level dimensions of information privacy. Within each dimensional view a further three sub-classifications were proposed each with their own unique nature.

Originality: This paper provides an Information Privacy taxonomy for Collaborative Environments, the first of its kind to be proposed. A number of new Information Privacy terms are defined that make up the categorization and classification of Information Privacy concepts and components.
\end{abstract}


Keywords: Information Privacy, Taxonomy, Meta Privacy, Data Protection.

Paper Category: Research, Conceptual, and Viewpoint.

\section{Introduction}

By definition, taxonomy is '... A scheme that partitions a body of knowledge and defines the relationships among the pieces. It is used for classifying and understanding the body of knowledge.' (IEEE, 1986). As a field of research grows there comes a point in time that the subject matter should be at least theoretically classified into its bases, principles, procedures and rules. The area of Information Privacy, in particular, in the expanding field of Collaborative Environments, is in need of such an arrangement. This paper addresses this issue by providing an general introduction of overview of a taxonomy for Information Privacy in Collaborative Environments. The full taxonomy has not been included due to space limitations, but a much more detailed and expanded body of work has been produced by the authors of this paper.

The paper does provide a high level of detail and knowledge suitable for taxonomy of Information Privacy. Focus has been placed on Collaborative Environments (C.E.'s) due to their inherent data sharing nature and the related privacy issues they create. With the use of C.E.'s in many areas, including the health and intelligence sectors, there are numerous personal data privacy problems to address. Following on from the introduction, section 2 provides a background on the area of Information Privacy. The information privacy issues in Collaborative Environments are discussed in greater detail in Section 3. Section 4 provides the taxonomy proposal and its three key dimensions, followed by a conclusion in Section 5, and References at the end.

\section{Background and Related Work}

Before continuing it seems that no privacy proposal is complete without some mention of the 'type' of privacy, one is addressing. This is especially important when the subject of importance is a taxonomy. From a definition of a particular dimension of privacy one can loosely categorize the solutions aimed at each of them. Privacy in general is very subjective and means different things to different people. Common among all interpretations is the perspective that privacy is a human right but is context and environmentally dependent. A number of common privacy dimensions have been defined that have gained wide acceptance (Clarke, 1999a). They are as follows:

- Privacy of the person

- Privacy of personal behaviour

- Privacy of personal communications 
- $\quad$ Privacy of personal data

Personal data, also referred to as information privacy is the focus of this taxonomy. In (Clarke, 1999) Clarke also provides a well referenced definition of information privacy after initially stating it as being a combination of personal communication privacy and personal data privacy. His formal definition of information privacy is “... the interest an individual has in controlling, or at least significantly influencing, the handling of data about themselves." (Clarke, 1999). The Common Criteria (CC Org., 2005) provides a more formal requirements based definition for providing "... user protection against discovery and misuse of identity by other users.". As you can see from the CC definition, it is information systems requirements focused, with emphasis on identity protection. Identity protection is a major component of information privacy but by no means represents the complete embodiment of its full meaning.

The Platform for Privacy Preferences (P3P) of W3C (2002) is a significant effort to enable web and potentially information system users to gain control over their private information. Open to much debate as to whether it is truly a PET; it does provide automated notice and privacy policy reading from user web browsers. P3P has generated a lot of interest and naturally a lot of research and work material in the area. The scope of which is beyond this paper. What is of interest is its use of metadata to represent privacy policy settings of entities to further enhance their privacy protection. P3P can be used as an assurance mechanism for an entity to confirm the privacy policy preferences in a settings matching process. Metadata tags and document structures are used to store an entity's privacy settings and preferences. The entity requesting personal information also uses the metadata tags and document structures to represent their privacy policies and operational procedures.

A number of IBM Research groups and collaborators have been working on a few interesting approaches to privacy. Part of their work has been extensions and usage of P3P. This includes implementing P3P using Database Technologies (Argrawal et. all, 2003). This is an architectural alternative for implementing P3P, moving away from a client-centric model. Rather, it is based on a server-centric implementation that reuses database querying technology. The other P3P related work is termed the Platform for Enterprise Privacy Practices (E-P3P) (Karjoth et. all, 2002a). It defines technology for privacy-enabled management and exchange of customer data. The basic concept is to place access restrictions on personal data. The restrictions are expressed in a privacy-specific access control language. The Enterprise Privacy Authorization Language (EPAL) is another privacy initiative developed by IBM (Karjoth et. all, 2002b), (Backes et. all, 2003), (Backes et. all, 2004), (IBM, 2001). EPAL enables an organization to formalize the exact privacy policy that shall be enforced within the organization. It formalizes the privacy promises into policies and associates a consented policy to each piece of collected and possibly shared data. This is some what similar to our approach within a collaborative environment. In that the personal data is stored along with the privacy conditions agreed to, and customized, by the user at the time of data collection and sharing. The privacy information attached to the personal data elements is used to determine access controls and privileges associated with separate users and roles within the collaborative environment.

A number of privacy enhancing tools and contributions have been provided by David Chaum. In particular his work on: Blind Signatures (Chaum, 1985a), (Chaum, 
1992), (Chaum, 1985b); Digital Cash (Chaum, 1989); Un-traceable electronic mail, return address, and digital pseudo-nyms (Chaum, 1981); and privacy protecting protocols for transmitting personal information between organizations (Chaum, 1986). Of most interest to our research has been the work on transmission of personal information, specifically its application to information sharing and transmission in collaborative environments. Again due to space limitations interested readers are directed to references provided for further consultation.

Another area of related work is research being done on privacy through design particularly in Ubiquitous Systems. Privacy by design has guided our own solution and is an approach we feel is crucial to comprehensive privacy protection through out a collaborative environment (Boyd et. all, 2002). Publications in the area include Privacy Design Principles used by various governments and organizations (Govt. of Ontario, 2000), (U.S. Dept. of Justice, 2000), (Hope-Tindal, 2001), 'Design for Privacy in Ubiquitous Computing Environments' (Schwartz, 1999), and 'Preserving Privacy in Awareness Systems' (Patil and Kobsa, 2004). Other important literature contributions in relation to privacy in Ubiquitous Computing Environments include (W3C, 2002), (Duan and Canny, 2004), (Goecks and Mynatt, 2002), (Langheinrich, 2002), (Langheinrich, 2001) each of which has provide additional inspiration for our own work.

\section{Information Privacy Requirements for Collaborative Environments}

Collaborative environments present major challenges to privacy. This fact is highlighted by Duan and Canny (2004) where they state: "By its nature, collaboration involves the exchange of information between collaborators. It may also involve automation ...". There is a need to establish a balance between the benefits collaborative environments provide for knowledge discovery and sharing against the protection of individual and organizational privacy needs. In many countries now and in the immediate future privacy protection is enforceable by a number of laws and regulations. Privacy is no longer an issue for ad-hoc consideration or as an after thought once a system has been implemented. This is especially so in Collaborative Environments, due to their increased sensitivity and potential risks to privacy. Organizations and users need better control over how people gain access to each other and their personal information (Bellotti, 1996).

While privacy is a popular subject in relation to Collaborative Environment literature it has not been adequately examined in its own right. Rather, is has largely been addressed as an issue of security by system designers (Goecks and Mynatt, 2002). From what we are aware there has been no substantial amount of research done into privacy protection in Collaborative Industrial Environments. This paper, and further more extensive work by the authors, provides an introduction and overview of a taxonomy for information privacy in collaborative environments (C.E.'s). The aim is to provide an organized approach to information privacy in C.E.'s. Provid- 
ing clear classifications and categorizations of the many components and dimensions present for future knowledge and understanding in the area.

\section{Overview of Information Privacy Taxonomy}

We have identified three dimensions that make up the highest level of the information privacy taxonomy. These dimensions are time, matter, and space. All are interrelated and have different influences over information privacy. The dimensions translate to three corresponding views of information privacy within a collaborative environment. The views are Computation View, Content View, and Structural View respectively. Each of the dimension and corresponding views are discussed in more detail in the following sub-sections.

\section{"TAKE IN FIGURE 1"}

Fig. 1. Three dimensional representations of Collaborative Environments' Information Privacy Taxonomy Nature Classifications.

\section{Dimension 1: Computation View}

The time dimension, and therefore the computation view, reflects the level of privacy protection. Its time relevance relates to the amount of time and resources required to compromise the stated level of privacy protection. Three categories of privacy protection have been defined, each classified from the highest level of protection to the lowest. The three include Ideal Privacy, Computational Privacy, and Fragile Privacy, listed from highest to lowest protection respectively.

NATURE $(\mathrm{N})=$ Level of Privacy Protection $(\mathrm{pp})$.

\section{Ideal Privacy}

Privacy Protection: $\mathrm{pp}=\infty$ for Ideal Privacy

With Ideal Privacy, users at all times determine when, how and what personal information is revealed. Additionally personal data owners decide to what extent others can utilize their information once access is granted. Ideal Privacy gives users complete control over their personal data and more generally all of their information privacy concerns.

Definition 1: Ideal Privacy provides the highest level of privacy protection (theoretical and practical), providing users with complete control over all of their privacy concerns. No amount of computation can compromise ideal privacy protection.

$$
\{\text { As } \mathrm{t}->\infty \text { AND } \mathrm{r}->\infty ; \mathrm{pp}=\infty\} \text {. }
$$


This translates to: Given an infinite amount of time $(t)(t->\infty)$ and unlimited computational resources $(\mathrm{r})(\mathrm{r}->\infty)$ privacy protection $(\mathrm{pp})$ will always remain at the highest level and stay uncompromised $(\mathrm{pp}=\infty)$.

\section{Computational Privacy}

Privacy Protection: pp -> 0 for Computational Privacy

With Computational Privacy, users are provided with significant control over when, how and what personal information is revealed. Additionally personal data owners are the primary entities deciding to what extent others can utilize their information once access is granted. Computational Privacy gives users a high level of control over their personal data and more generally all of their information privacy concerns. However, system owners and data collectors also have a level of control over personal data collection and use, once terms have been agreed upon with personal data owners. Computational Privacy means that it is infeasible to compromise privacy protection within reasonable operational parameters. However, given a very long amount of time and a very large amount of resources, it may be possible to compromise the level privacy protection.

Definition 2: Computational Privacy provides a medium or operational level of privacy protection, providing users with significant but not complete control over all of their privacy concerns. With an infinite or unreasonably large amount of computation, computational privacy protection can be compromised.

$$
\{\text { As t- }>\infty \text { AND r- }>\infty ; \text { pp }->0\} \text {. }
$$

This translates to: Given an infinite amount of time (t) $(\mathrm{t}->\infty)$ and unlimited computational resources $(\mathrm{r})(\mathrm{r}->\infty)$ privacy protection $(\mathrm{pp})$ will eventually be compromised (pp $->0)$.

\section{Fragile Privacy}

Privacy Protection: pp ->> 0 for Fragile Privacy

Given a reasonable amount of time and resources fragile privacy can be compromised. This level of privacy protection is only deemed effective against weak threats and attacks. Unfortunately, a large number of collaborative environments (C.E.'s) are of this nature, when they should be offering higher levels of privacy protection. As the adaptation and uses for C.E.'s have increased so has the need for better privacy protection. Many internet sites are still of the format that an entity either accepts the organizations stated privacy as is, or the entity is denied access to their services and resources. Additionally, it is normally the case that if the entities consent is given, control over most personal data is relinquished to the information collectors. What further exacerbates the problem is that for the majority of entities, they do not really pay attention to the finer details of the privacy policy they are agreeing to. This results in a privacy agreement that is very fragile in its nature and understanding. Either 
the entity had no choice but to agree to the conditions, or they did not understand what they were agreeing to.

Definition 3: Fragile Privacy provides the lowest level of privacy protection, providing users with limited control over all of their privacy concerns. With a reasonable amount of computation, fragile privacy protection can be compromised.

$$
\left\{\text { As } \mathrm{t}->\mathrm{N}_{\mathrm{t}} \text { AND r- }>\mathrm{N}_{\mathrm{r}} ; \mathrm{pp}=0\right\} \text {. }
$$

This translates to: Given a reasonable amount $\left(\mathrm{N}_{t}\right.$ a large value) of time (t) $\left(t->N_{t}\right)$ and a reasonable amount $\left(\mathrm{N}_{\mathrm{r}}\right.$ a large value) of computational resources $(\mathrm{r})\left(\mathrm{r}->\mathrm{N}_{\mathrm{r}}\right)$ privacy protection $(\mathrm{pp})$ will be compromised $(\mathrm{pp}=0)$.

\section{Dimension 2: Content View}

The matter dimension, and therefore the content view, reflects the privacy of collaborative environment objects. Its matter relevance relates to the different types of data that require privacy. Three categories of objects have been defined and each classified accordingly. The three include Data Privacy, Identity Privacy, and Meta Privacy.

NATURE $(\mathrm{N})=$ Objects Privacy $(\mathrm{Obj})$.

\section{Data Privacy}

Object: Data $->$ knowledge and information $->$ \{Data, Text, Emails, Documents, Files, Logs, Transcripts, etc\}

Data privacy is the protection of an entities personal data that is being collected, shared, and stored. Ideally the protection is complimented with the entity having complete control over their personal data. There are a number of formal definitions for data privacy that are useful in our taxonomy representation. One such definition refers to data privacy as the '... evolving relationship between technology and the legal right to, or public expectation of privacy in the collection and sharing of data.' (Clarke, 2002). The problem in virtual collaborations is that they are environments made up of interconnected heterogeneous information systems that have different privacy rules and laws governing their operation.

Therefore, those tasked with the management and administration of virtual collaborations must take the responsibility for securing personal data and monitoring its secure use. Further, the control and management of an entity's personal data, collected in a collaborative environment, should be tasked to that data owner. This approach has the added benefit of distributing the privacy protection responsibilities between system users and owners. Not only are data owners responsible for their own data but they have the desired control over what data is accessible, by who and for what specific purposes it can be used. Using this method also conforms to most privacy policies and legislations either in use or being introduced in many organizations. 


\section{Identity Privacy}

Object: Entities -> individuals, groups, and organizations -> \{Identity, Identification, Authentication

One of the top five privacy issues for the year 2005 was Identity Management, specifically the need to balance privacy and security in such a context (Ponemon, 2004). The same report states 'Identity is key to protecting personal information and privacy rights'. In an information system and in a broader virtual collaboration an identity is a set of data about an entity (individual, group, or organization) that could be used to differentiate them from other entities in the same environment.

Therefore, identity protection involves securing an entity's identity from unwanted disclosure or discovery. From a privacy preserving perspective, identity protection is concerned with entity control over how they interact with the information system and other entities within the system. Entities should have a choice as to when, how and to who they reveal their identity to and also who has access to it. Further, transactions and interactions should be able to be carried out in any of the following ways:

- Identifiable: the ability to differentiate an entity or transaction from a group of other entities or transactions.

- Pseudo-Anonymous: the inability to differentiate an entity or transaction from a group of other entities or transactions in the normal course of events.

- Anonymous: the total inability to differentiate an entity or transaction from a group of other entities or transactions.

It is also possible for a single entity to have multiple pseudo-anonymous identities, also referred to as 'nyms' (van Blarkom et. all, 2003). Likewise, in an ideal setting it should also be possible for many entities to use the same 'nym'. In this papers context this would allow all members of a group or organization to use the single 'nym'. Individuals using the same 'nym' would not allow true identity protection. That is, unless the entities had consented to absolute disclosure and control over each others personal data. When using multiple identities it should not be possible for other entities to deduce that any two identities represent the same entity. That is, there should be no way that a relationship can be established or shown between any two system pseudo-anonymous or anonymous identities. This includes past, present and future system and processing data, actions, and behaviours.

The use of pseudo-anonymous and anonymous identities is often in conflict with a long held misconception that a person's true identity needs to be known for authenticated access to information systems and their resources. It is now widely accepted that is most cases a suitable pseudo-anonymous identity is just effective for the majority of authorization techniques. That is, it is possible to authorize access by a form of identity rather than authenticating access through an entities true identity. Therefore, the three key aspects to identity privacy are (Clarke, 1999b):

- Identity

- Identification

- Authentication 
There is a complex relationship between all of the aspects, especially from an information systems privacy and security perspective. Further discussion of this subject is beyond the scope of this paper due to space limitations.

\section{Meta Privacy}

Object: Metadata and Metastructure Information -> Approaches and Purpose: -> (1) Metadata and Metastructure Content: \{Personal Privacy Protection versus Privacy Policy and Preferences Representation $\}$; (2) Unlinkable and Unobservable -> \{Risks versus Benefits\}.

Meta Privacy is a relatively new term, first formally defined in (Skinner et. all, 2005). A common definition for the word Meta is as a prefix used in an information systems context as meaning "relating to" or "based on". More formally it is a prefix meaning "information about". So when used in conjunction with the term privacy, to formulate the term Meta Privacy, it means information about privacy. Meta privacy is concerned with the information used to support other system services and processors that impact upon an entities privacy. An entity may be an individual, group, or organization. Meta Privacy encompasses the use and management of metadata and metastructure information. It is the metadata and its implementation details, metastructure, which can be the source of either privacy enhancing benefits or privacy invasive drawbacks. This is determined by metadata use, such as P3P [Above], or abuse.

Meta Privacy is defined by the following definition: 'Meta Privacy means ensuring the security and privacy of data about privacy and personal data. Meta privacy is concerned with the security and privacy of the information used to support other system services and processors that may impact upon an entities privacy. This encompasses the protection of metadata and metastructure information that may reveal an entities identity and other personal information' [32].

The Metastructure components are composed of the data concerned with the functioning and structural details of the information systems and their many components. This may include information on the access controls used in the systems, the system and policy frameworks which supplies rules regarding the relationships within and between the systems and their policies, and other information about the system and component structures and the interoperation. When dealing with information systems and more generally collaborative environments the management of metadata and metastructure information involves serious privacy considerations.

The controlled use, access to, and storage of metadata and metastructure information must be guided by stringent privacy protection procedures. It is the metadata and its implementation that can be the source of either privacy enhancing benefits or privacy invasive drawbacks. This applies also to the use of metastructure information. Both types need to be protected and is the focus of this sub-section. 


\section{Dimension 3: Structural View}

The space dimension, and therefore the structural view, reflects the privacy of collaborative environment entities. Its space relevance relates to the different types privacy applied to various entities and relationships within the Collaborative Environment. Three categories of entities have been defined and each classified accordingly. The three include Individual Privacy, Group Privacy, and Organizational Privacy.

NATURE $(\mathrm{N})=$ Privacy of Entities and Relationships $(\mathrm{R} \ln )$.

\section{Individual Privacy}

Individual Privacy is the privacy of an individual entity. In this paper the context of use refers an entity or user within the Collaborative Environment. It is an entity that has provided personal data that they wish to remain private or protected for privacy reasons. At the most fundamental level each individual entity that is a member of the collaborative environment (C.E.) should be entitled to privacy protection. This encompasses any personally identifiable information (PII) they have provided during registration and ongoing membership of the C.E.. If an entities personal data is revealed without their permission, this would constitute a privacy breach. Individual privacy includes protecting both personal data and related metadata and metastructure information. The protection supports the concept of unobservability.

An individuals perception of privacy is highly subjective and context specific. Therefore, what constitutes privacy protection in the C.E. should be clearly stated in the associated privacy policy and operational procedures. Anonymous and pseudoanonymous identities should be available for use by individual entities when ever possible. The use of multiple identities of this type should also be available to support unlinkability.

Definition 4: Individual Privacy is concerned with the protection and preservation of an individual entity's privacy.

Individual privacy protects each user from undesirable intrusions and the maintenance of their personal space. In an information privacy context it means that a user's personal information is protected from unauthorized access and use. As individuals may be members of a number of groups, and organizations they are able to establish both Committed and Not-Committed privacy relationship.

$$
\{1->1(! \varepsilon \| \varepsilon) ; 1->\mathrm{n}(! \varepsilon \| \varepsilon) ; \mathrm{m}->\mathrm{n}(! \varepsilon \| \varepsilon): \text { where } \mathrm{m}=\mathrm{n} \| \mathrm{m} \neq \mathrm{n}\} \text {. }
$$

(Note: ! $\varepsilon$ means Not-Committed and $\varepsilon$ means Committed)

This states that within a Collaborative Environment (C.E.) Individual Privacy is maintained for any entity member. The entities provided privacy protection may be a single entity, one entity to $n$ entities, or even $m$ to $n$ entities with the C.E.. The entities may be either committed or not-committed to their own privacy protection, provided by the C.E.. 


\section{Group Privacy}

Groups in this context are those with no entity commitments. They are often dynamic and ad-hoc groupings in nature as a result. The members of the groups and the groups themselves need their own levels of privacy protection depending on the needs of the membership and group. These needs are also influenced by the reason for group existence and the data the group handles and produces. When members join and leave the group there needs to be at least Fragile Privacy protection maintained over the group and entity members data.

Within a virtual collaboration there may be any number of formal and informal groups. Each of these has their own set of privacy concerns that must be addressed. A group, like an individual, produces 'personal' information that should be under the management of the group. The group controls who, when, where and what information is revealed to other entities. Depending on the nature of the group, formal or informal, there are different levels of privacy sensitivity to the group for different members. Membership is also dependant on other factors including time, roles, requirements and personal needs.

Definition 5: Group Privacy is concerned with the protection and preservation of a Group's and each Non-Committed individual group member's privacy.

Group privacy protects the personal information of the group and each member in the group. The privacy protection is provided regardless of the non-committed nature of the group members. Groups may be ad-hoc, dynamic and time dependant relations. Group Privacy aims to support the varying non-committal nature of group member-

ships. This means that Group Privacy ensures that during the formation, duration, and after they have been dispersed the privacy of the group and each entity member of the group is protected.

$$
\{1->1(! \varepsilon) ; 1->\mathrm{n}(! \varepsilon) ; \mathrm{m}->\mathrm{n}(! \varepsilon): \text { where } \mathrm{m}=\mathrm{n} \| \mathrm{m} \neq \mathrm{n}\} .
$$

(Note: ! $\varepsilon$ means Not-Committed)

This states that within Collaborative Environments (C.E.'s) Group Privacy is maintained for any group and group member. The entities provided privacy protection may be a group with single membership, a group with one entity to $\mathrm{n}$ entities, or a group with $m$ to $n$ entities. The entities are not-committed to the groups privacy provided. This is as a result of the Group being defined by ad-hoc, informal and noncommitted entity membership.

\section{Organizational Privacy}

Organizations in this context are those with formal and informal commitments required by their entity membership. They are often structured, planned, and governed by a set policies and procedures. The members of an organization are committed to privacy. The privacy is maintained with when members join and leave the organization. Within a Collaborative Environment (C.E.) there may be any number of formal and informal organizations. Each of these has their own set of privacy concerns that must be addressed. An organization, like an individual and group, also produces 'per- 
sonal' information that should be under the management of the organization and afforded privacy protection. The organization controls who, when, where and what information is revealed to other entities. Depending on the nature of the organization, formal or informal, there are different levels of privacy sensitivity to the organization for the different entity members. Membership is also dependant on other factors including time, roles, requirements and personal needs. Organizations also have a number of additional privacy issues that need to be managed. This includes the changing members of the group and ensuring privacy policies are still adhered to. What's more, this is even after organizational membership has been relinquished.

Definition 6: Organizational Privacy is concerned with the protection and preservation of an Organization's and each Committed individual organizational member's privacy.

Organizational privacy protects the sensitive personal information and activities of an organization, in addition to the personal information of the organizational members. Individual entities are committed to organizational privacy protection as well as their own and each entity within the organization. Organizations generally have more stable and committed memberships that do groups. This allows formal individual commitments, privacy policy development, and privacy focused operating guidelines.

$$
\{1->1(\varepsilon) ; 1->n(\varepsilon) ; \mathrm{m}->\mathrm{n}(\varepsilon) \text { : where } \mathrm{m}=\mathrm{n} \text { OR } \mathrm{m} \neq \mathrm{n}\} \text {. }
$$

(Note: $\varepsilon$ means Committed)

This states that within the Collaborative Environment (C.E.) Organizational Privacy is maintained for any organization and organizational member. The entities provided privacy protection may be an organization with single membership, an organization with one entity to $n$ entities, or an organization with $m$ to $n$ entities. The entities are committed to the organizational privacy provided. This is as a result of the Organization being defined by committed entity membership often governed by set privacy policies, guidelines and operating procedures.

\section{Taxonomy Hierarchical Organization}

Taxonomy means the arrangement, organization, and classification of some fields or specific subject matters content and concepts. Fig. 2 below provides a graphical representation of the hierarchical organization of the Information Privacy Taxonomy for Collaborative Environments. The root of the tree starts with a general node representation of Information Privacy. The second level corresponds to the three dimension and corresponding views. The third level on the diagram encompasses the three key components within each dimension.

\section{"TAKE IN FIGURE 2"}

Fig. 2. Hierarchical organization of an Information Privacy Taxonomy. 


\section{Conclusion}

Privacy, and more specifically, Information Privacy, has been gaining research momentum of late. The increased interest in the field has generated numerous new definitions, terminologies, and proposed solutions to the complex privacy issues. With the increasing abundance of material in the field it has become difficult to define and classify information privacy components. This paper has addressed this issue by providing an Information Privacy taxonomy. The contribution proposed has been limited by the space restrictions of the paper, but has provided a unique arrangement of many of the important information privacy components and dimensions.

Focusing the taxonomy on a Collaborative Environment Information Privacy issues has highlighted three key dimensions of the taxonomy. The dimensions are the Computation View, the Content View, and the Structural View. Within each there are additional categorizations to clearly define each of the dimensions and elements contained within them. The Computation View had three sub-classifications of Ideal Privacy, Computational Privacy, and Fragile Privacy as they relate to the level of Privacy Protection. The content view also had three sub-categories of Data Privacy, Identity Privacy, and Meta Privacy as they relate to objects. Finally, the Structural View likewise has three sub-categories of Individual Privacy, Group Privacy, and Organizational Privacy as they relate to entities and their privacy relationships.

\section{References}

Agrawal, R., Kiernan, J., Srikant, R., and Xu, Y. (2003), 'Implementing P3P Using Database Technology'. 19th International Conference on Data Engineering, Bangalore, India.

Backes, M., Pfitzmann, B., and Schunter, M. (2003), 'A Toolkit for Managing Enterprise Privacy Policies'. ESORICS 2003, LNCS 2808, pages 162-180, October.

Backes, M., Bagga, W., Karjoth, G., and Schunter, M. (2004), 'Efficient Comparison of Enterprise Privacy Policies'. SAC'04, Nicosia, Cyprus, March.

Bellotti, V. (1996), 'What You Don't Know Can Hurt You: Privacy in Collaborative Computing'. BCS HCI 1996: 241-261.

Boyd, D., Jensen, C., Lederer, S., and Nguyen, D.H. (2002), 'Privacy in Digital Environments: Empowering Users'. CSCW 2002 workshop, New Orleans, 16 November.

Common Criteria (2004), 'Common Criteria for Information Technology Evaluation'. http:// www.commoncriteria.org, January.

Chaum, D. (1985a), 'Showing Credentials Without Identification. Signature Transfers Between Unconditionally Unlikable Pseudonyms'. Advances in Cryptology - EUROCRYPT '85, Proceedings; LNCS 219; Springer Verlag, pages 241-244.

Chaum, D. (1992), ‘Achieving Electronic Privacy’. Scientific American, August 1992, p. 96101. 
Chaum, D. (1985b), 'Security without Identification: Card Computers to make Big Brother Obsolete'. Communications of the ACM, 28, 10, Oct..

Chaum, D. (1989), 'Privacy Protected Payments: Unconditional Payer and/or Payee Untraceabiliy'. Smart Card 2000: 69-93.

Chaum, D. (1981), 'Untraceable Electronic Mail, Return Ad-dresses, and Digital Pseudonyms'. Communications of the ACM, 24,2, Feb..

Chaum, D. and Evertse, J.H. (1986), 'A Secure and Privacy-Protecting Protocol For Transmitting Personal Information Between Organizations'. Crypto '86, LNCS 263, Springer-Verlag, Berlin 1987, 118-167.

Clarke, R. (1999a), 'Introduction to Dataveillance and Information Privacy, and Definitions and Terms.'. http://www.anu.edu.au/people/Roger.Clarke/DV/Intro.html.

Clarke, R. (2002), 'Introducing PITs and PETs: Technologies Affecting Privacy'. Xamax Consultancy.

Clarke, R. (1999b), 'The Real 'Who's Who' of the Electronic Commerce: The Identification of Organizations.'. Xamax Consultancy. Prepared for Journal of Strategic Information Systems. http://www.anu.edu.au/people/Roger.Clarke/DV/Intro.html.

Duan, Y. and Canny, J. (2004), 'Protecting User Data in Ubiquitous Computing: Towards Trustworthy Environments'. Privacy-Enhancing Technologies (PET) 2004, Toronto, CA, May.

Goecks, J. and Mynatt E. (2002), 'Enabling Privacy Management in Ubiquitous Computing Environments through Trust and Reputation Systems'. CSCW 2002 workshop, New Orleans, 16 November.

Government of Ontario (2000), 'Privacy Design Principles - Personal Information'. http://www.gov.on.ca/MBS/english/fip/pub/pdp.html, 23 May.

Hope-Tindal, P. (2001), 'Managing Privacy and Security Risks Through Architecture Design: An Enterprise Privacy View'. Data Privacy Partners, November.

IBM (2003), Enterprise Privacy Authorization Language (EPAL) Specification. http://www.zurich.ibm.com/security/enterprise-privacy/epal/.

The Institute of Electrical and Electronics Engineers Inc (1987), "Standard Taxonomy for Software Engineering Standards (ANSI)". IEEE Standard 1002-1987.

Karjoth, G., Schunter, M., and Waidner, M. (2002), 'Platform for Enterprise Privacy Practices: Privacy-enabled Management of Customer Data'. 2nd Workshop on Privacy Enhancing Technologies, LNCS. Springer Verlag.

Karjoth, G. and Schunter, M. (2002), 'A Privacy Model Enterprises'. 15th IEEE Computer Security Foundations Workshop, June. 
Langheinrich, M. (2002), 'Privacy Invasions in Ubiquitous Computing'. Privacy in Ubicomp 2002, GÖTEBORG, Sweden.

M. Langheinrich (2001), 'Privacy by Design - Principles of Privacy Aware Ubiquitous Systems'. Ubicomp 2001, Atlanta.

Patil, S. and Kobsa, A. (2004), 'Preserving Privacy in Awareness Systems'. Wissen in Aktion.

Ponemon, L. (2004), ‘Top 5 Privacy Issues for 2005'. Computerworld, Dec 28th.

Schwartz, P.M. (1999), 'Privacy and Democracy in Cyberspace'. 52 VAND. L. REV. 1609, 1610-11.

Office of the Ontario Information and Privacy Commissioner and the United States Department of Justice (2000), 'Privacy Design Principles for an Integrated Justice System". http://www.ojp.usdoj.gov/archive/topics/integratedjustice/pdpapril.htm. Working Paper.

Skinner, G., Han, S., and Chang, E. (2005), 'Defining and Protecting Meta Privacy: A New Conceptual Framework Within Information Privacy.. International Conference on Computational Intelligence and Security, Xian, China, Dec 15-19.

van Blarkom, G.W., Borking, J.J., and Olk, J.G.E. (2003), 'Handbook of Privacy and PrivacyEnhancing Technologies'. PISA Consortium, The Hague.

W3C (2002), 'W3C: The platform for privacy preferences 1.0 (P3P1.0) specification'. W3C Proposed Recommendation, http://www.w3.org/TR/P3P. 